\title{
The Application of Computer Technology in Mongolian College English Teaching
}

\author{
https://doi.org/10.3991/ijet.v12i02.6044 \\ Xiaojun Zhao \\ Inner Mongolia Normal University \\ xiaojun.zhao@hotmail.com
}

\begin{abstract}
Based on the demand of modern teaching, this paper researches the application of and problems faced by Mongolian information processing and computer multimedia technology in computer-assisted instruction. It also sets the teaching software content and learning goal and further designs the teaching software according to the English learning features and characteristics of choosing the medium of information of Mongolian college students. This software is suitable for use in learning and teaching bilingualism second language to Mongolian students, and teachers and can realize the independent code of bilingual characters, Mongolian input and output, Mongolian character handling and bilingual inter-translation functions. Manufacture and development of bilingual teaching software facilitates the enthusiasm of Mongolian college students to learn English, improve the language learning environment and plays a positive facilitation role in multilanguage integration and cultural development of the Mongolian area.
\end{abstract}

Keywords - Computer-assisted, Inner Mongolian College Students, Multilanguage integration, Software design

\section{$1 \quad$ Introduction}

Although computer-assisted instruction in China started relatively late, it has developed rapidly in recent years. People have seen the obvious change of teaching efficiency after multimedia is introduced into the teaching [1]. As the need for teaching software is urgent, many areas and departments are especially devoted to the design of computer-assisted teaching software, while finished software completely comprised of complete teaching software is few [2]. The multimedia teaching software is especially suitable for the class teaching content, but is even fewer. In the English software, due to the influence from the exam-oriented education, modern education techniques are used to activate the teaching textbooks, and its application value is much less in the class teaching. The prominent problem is that the software does not exert the advantages of education techniques. Functions that can be simply realized with other equipment are re-designed by utilizing the computer.

Assisted Mongolian teaching software is difficult to find on the market. In the Mongolian area, Trilingual Multimedia Teaching Software of Mongolian, Chinese 
and English and Bilingual Multimedia Teaching Software of Mongolian and Chinese developed by Inner Mongolian Normal University and Mongolian Courseware Spirit developed by Ergun Company are commonly found. They have their own features [3]. In recent years, there also is other Mongolian courseware on the market and the manufacture of these courseware mostly do not realize the courseware of real sense. In brief, the main problem faced by the manufacture of Mongolian courseware is that the bottom-layer code of Mongolian writing is not transformed and the software cannot be directly used. In the manufacturing process, we must firstly make the special controls used for displaying and inputting the Mongolian characters and the manufacturing of multimedia assisted software on this basis [4].

\section{Software Development Teaching Content and User Character Analysis}

The purpose of multimedia-assisted English teaching software is to let the learner efficiently complete the learning of teaching content through the operation of software [5]. Therefore, developing multimedia-assisted teaching software must follow the general rule of media software development, and also must remain in accordance with the requirements of class teaching content and give consideration to the mental features of middle school students and other factors at the same time. In the software development, achieving the optimal combination requires analysis of the following factors.

\subsection{Determining teaching content and goal}

Analysis of the teaching content: English textbooks of four-year junior middle school out of a nine-year compulsory education are selected to be the courseware and teaching content. This includes the daily communication, orientation explication and courtesy language and is involved with change of nominative case and objective case of personal pronouns, use of antonyms, sentence patterns of daily dialogue and other concrete contents [6]. Words used for most common in daily life and the themes favored by the teenagers and children are chosen to be the text, which are relatively acceptable for the ability of children and teenagers. The learning focus of partial content is pronunciation and cultivating the language sense and English learning as a part of language learning. English teaching with multimedia measures is introduced with vivid pictures to imitate scenes of daily life, which more easily attracts the attention of students than the traditional class teaching, and through the interaction of students and computers, it lets the student review and consolidate the learnt knowledge according to their own concrete situation [7].

\subsection{Learner features analysis}

Analysis of the general features of the learner: General features of the learner refers to the mental and social features that influence the learner in learning the relevant 
subjects. Although they have no direct relation with the concrete subject content, they can influence the selection and organization of teaching lesson plans and on the learning content [8]. At the stage of middle school, a student's thinking ability, especially logical thinking develops rapidly, such as foresee-ability, so that the students can complete the self-preview and independent learning. Self-awareness or selfmonitoring capability becomes more obvious. As affective thinking of a student gradually transforms into rational thinking, students can independently complete a part of work, but they cannot accomplish the whole work with complete independence [9].

\section{Software System Design and Development}

According to foreign language teaching theory, we divide the software into the following modules: new classes, practices, tests, games and system maintenance. The module of new class is synchronous with the text to facilitate the class teaching of teachers or to use for the consolidation of students after class, including the through reading of text, through reading of single sentences and explanation and example sentences of key words. The module of practice completes the consolidation practice of the student system and practice to complete the forming type evaluation of the students. The module of tests makes the summary evaluation of the students. The module of games reviews the learned words and deepens the understanding and memory of these new words. The text provides the students with English songs for learning and appreciation divided by units [10].

Design of the unit content in every module is as follows: (1) Speech training at the introduction stage is carried out by centering on pronunciation and accent and is mainly practiced through words and sentences [11]. (2) Based on a specified quantity of words, the purpose of vocabulary exercises is to let the students learn the correlation among the words. (3) Grammar training includes some basic grammar points and a brief summary in each unit, focuses on grammar usage by giving typical sample sentences.

The design frame diagram of the software system is shown in Figure 1.

The "New class" portion of the software is the key link and is firstly introduced below:

\section{1 "New class" subsystem}

The "New class" subsystem is synchronous with the text content, including the pronunciation of whole or part of the dialogues, words inquiry and instant translation. The system structure drawing of this module is shown in Figure 2.

The users can choose the "learning content" in the "selection interface" while the accessible units include all units and the first lesson of each unit can be directly entered by clicking. Taking Unit 1 as the example, the user clicks on the Unit 1 button and enters into the first lesson of Unit 1, while the total course includes 65 lessons. The interface is shown in Figure 3. 


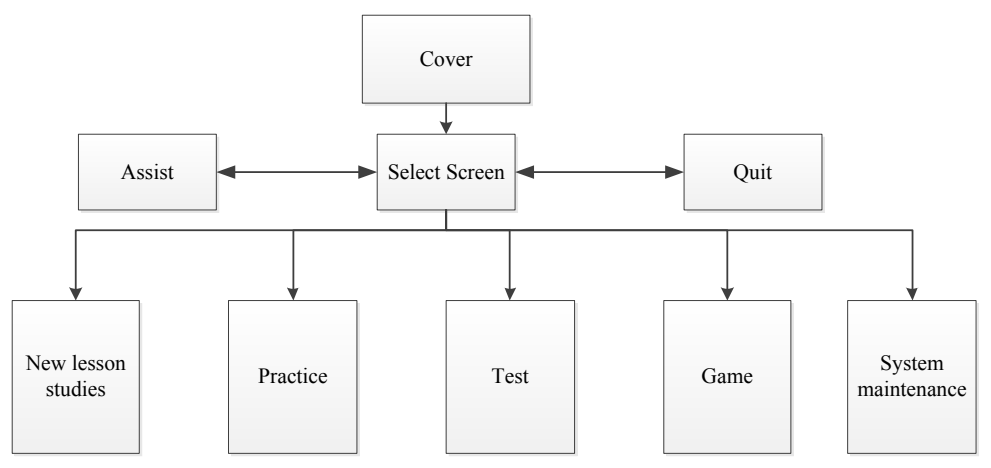

Fig. 1. The software system design frame

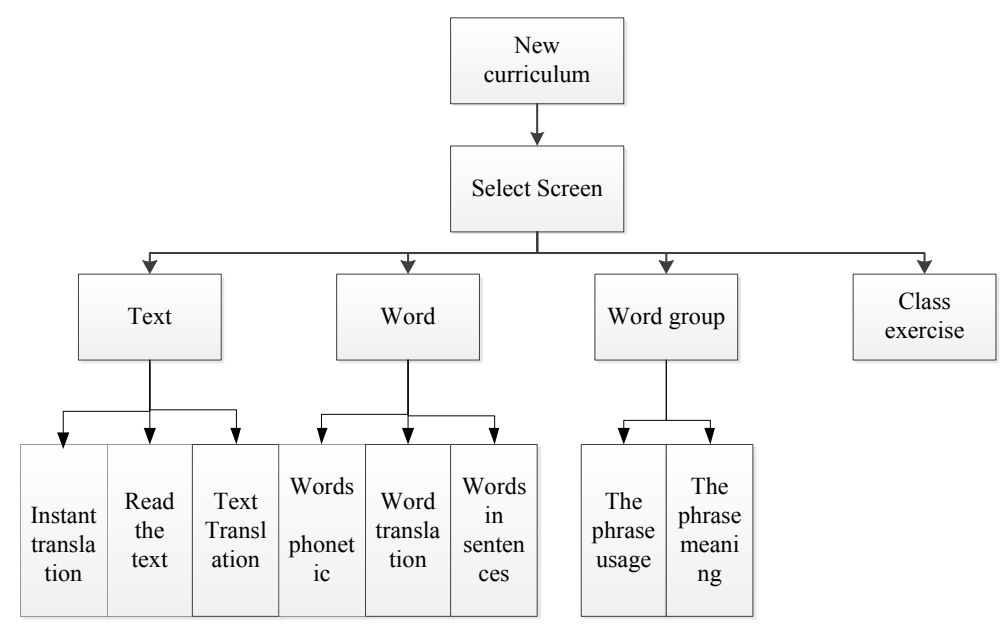

Fig. 2. New module of system structure

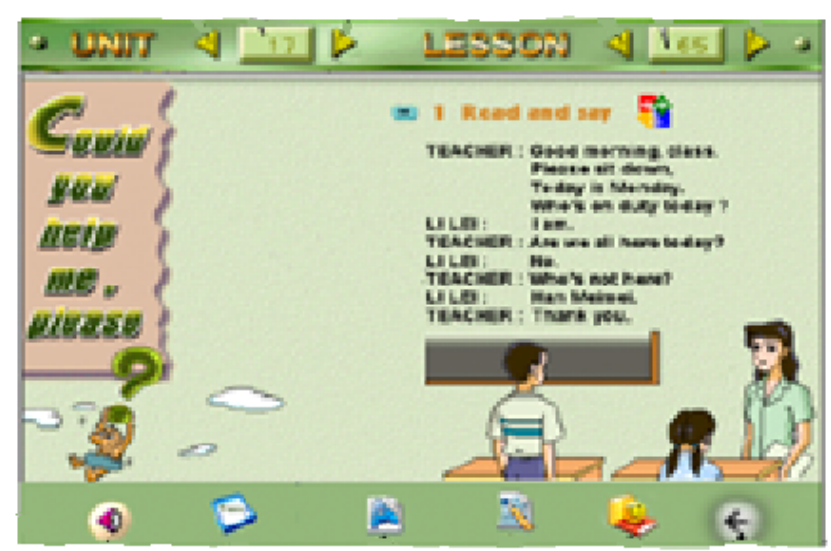

Fig. 3. Learning interface 
The functions of imaging buttons are given above in the indicator diagram. From the drawing, the design of interface applies the imaging design and the image is fresh and bright, which makes the teaching become flexible. The design is that the sounds and images appear in synchronous way to stimulate the hearing, vision, inference and multiple senses to perceive the new knowledge and make the students comprehend more quickly and makes the memory of that word or phrase more firm [12]. The button is horizontally placed at the bottom of the window which is familiar for most people. In the total module, the button location is relatively fixed and located at the bottom of window. The module arranges the button order according to the frequency of usage. For example, "reread" and "translate", which are frequently used in the module are placed at the front part, and the "practice", "test" and "game" buttons for connection are placed in a latter place. Meanwhile, they are logically divided, which is easy for the users to operate.

\subsection{Practice subsystem}

The practice subsystem re-appears with each topics of practice in a vivid way, thus deepening the understanding of the students about the learned content in class through practice [13]. Topics are closely connected with the knowledge points of each subject. The design of topic types is abundant and various. With the combination of features geared toward middle school students, the topic design is humanized. It attracts the attention of students as much as possible and makes them happily complete the practice in a fun way [14].

\subsection{Test subsystem}

The purpose of the test subsystem is to test the understanding and grasp of the new knowledge and new contents and check the degree of grasping of the students after the new class learning and practice learning subsystems. The test subsystem gives suitable suggestions after objectively evaluating the level of a student's understanding. For example, if the student does not grasp the content of one part well, the software will automatically give the relevant exercises or return to the corresponding practice modules to strengthen the knowledge of this part.

\section{Solution of Key Technology}

\subsection{Software development and realization}

Software realization takes the "form" as the basic unit and the order of the "form" introduction uses the sequence of "form" displayed in the program running as the criteria. 
1. As the introduction part of the program, the "cover" must be able to attract the attention of the student. The software uses animation to be the main content of "cover" and the animation is given in video format.

2. The "Selection" interface can choose the relevant learning module, including "new classes", "practices", "tests" and "system maintenance". Interface is in the form shown in the Figure 4. When the mouse passes the selection box, the selection box is displays a black button and the corresponding content can be entered by clicking on this button. This part mainly uses the mouse for monitoring, and when the mouse monitoring enters into the selection area, then the content of the relevant module is prompted by the voice.

3. By clicking on the "recording" button, a dialogue box pops up, as shown in Figure 5.

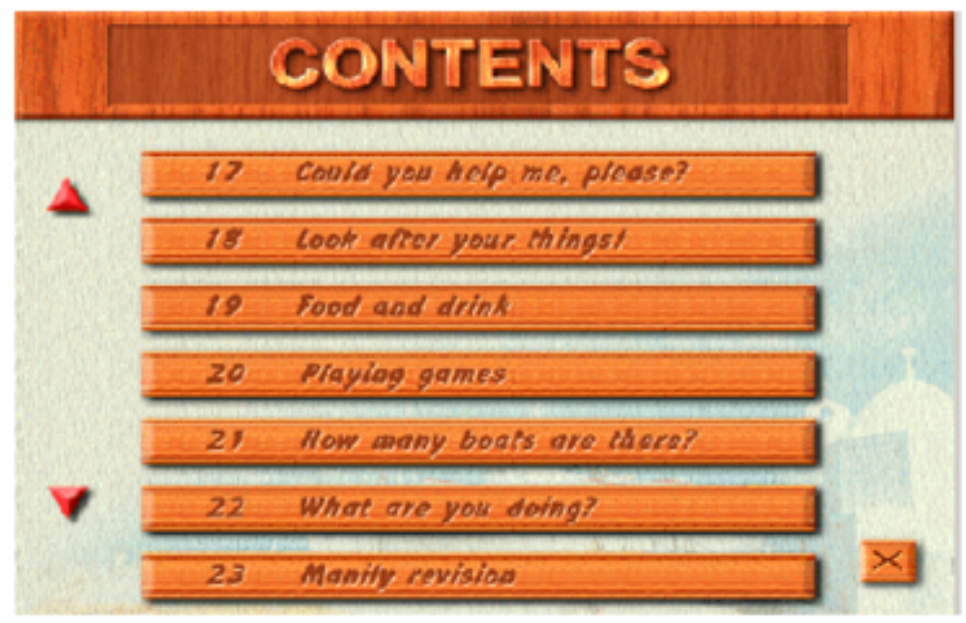

Fig. 4. Course selection interface

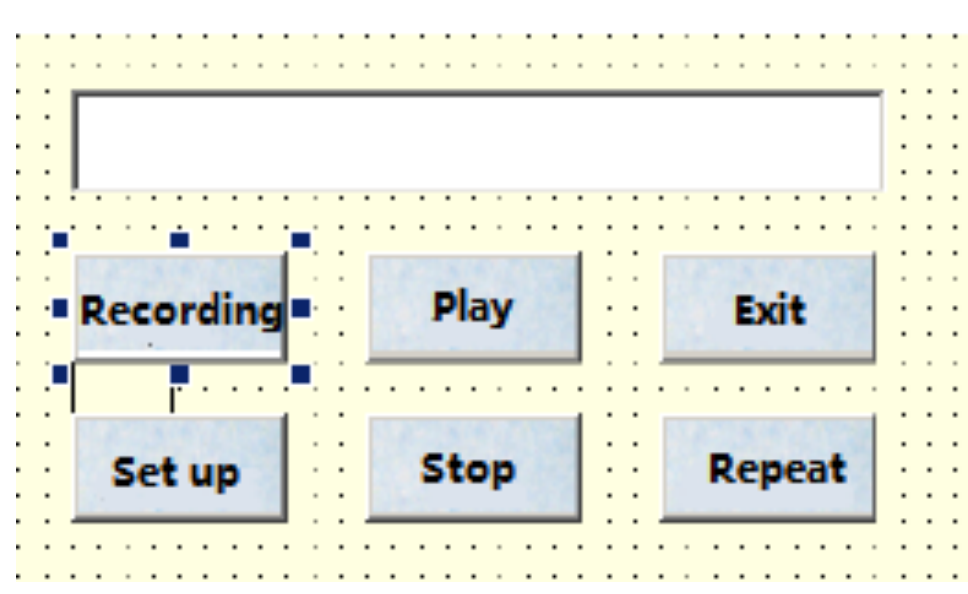

Fig. 5. Recording function interface 
4. The user enters the "course selection" interface by clicking on "new class". "Course selection" is used for the user to choose the text content which is suitable for him/her. The course selection is shown in Figure 4.

5. The "Text content learning" interface will be entered after selecting the relevant courses. This paper takes the content of Class 65, Unit 17 as an example to explain the text interface design and realization of its function.

In Figure 5, a recording can be made first and then the effect of the recording can be played. Recording and playing also uses the MM-Control. Its order includes "recording", "stop" and "play", and each function can be realized by clicking the button. The translation function can be opened by clicking the "translate" button. This function is accomplished with the indication of the mouse. The meaning of this paragraph for Mongolian statements can be displayed at the display interface of the window while the mouse indicates one paragraph of the text. "Practice" includes two parts: "practice in class" and "practice after class". "Practice in class" can be finished in class. Function buttons explained above are display in a graphic way to the users and the function's description and voice prompt description of the button start when the mouse stays for a while at the graphic area of a function button.

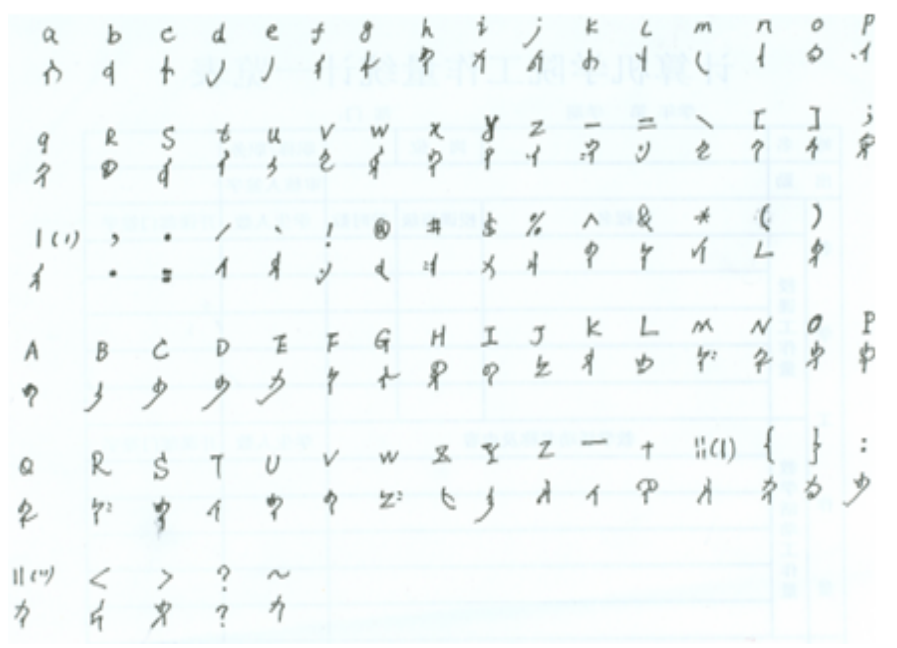

Fig. 6. Mongolian and English script comparison table

\subsection{Mongolian Design principle}

In the program design software, input of the verbal content relies on the "text control" and its display is achieved by relying on the "label control". Inputting and displaying the Mongolian script also uses the "text control" and "label control". Features of Mongolian control input and display complies with the writing convention of Mongolian input and display, and also conforms to the manufacturing principle of input and display control. The design of Mongolian control is reflected in the following four aspects: 
Paper-The Application of Computer Technology in Mongolian College English Teaching

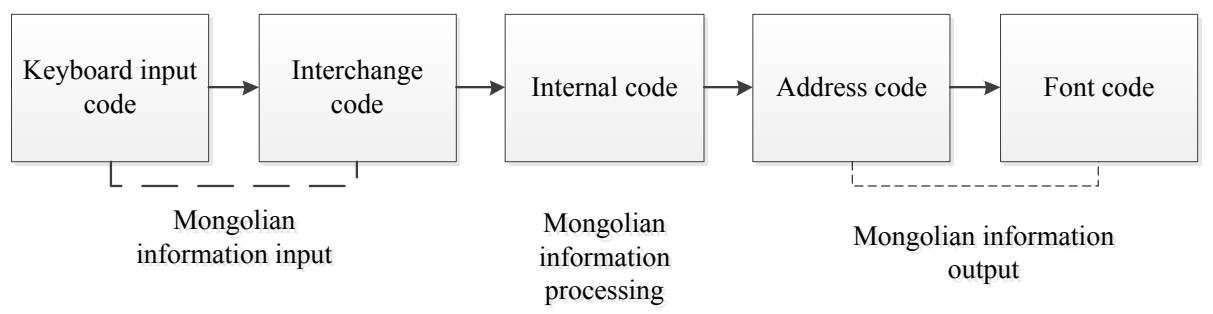

Fig. 7. Mongolian character processing flow chart

Mongolian code: As the ASCII code can only express the characters of English, however, it must also represent other languages and characters, such as Arabic, Chinese, Japanese and Mongolian, etc. [15], So expansion must be conducted. To display Chinese characters, China formulates the National standard code-GB231 code. Mongolian characters designed by the software are graphic characters and are stored in the blank code area of Chinese characters to finish the code work of Mongolian switch codes. Thus, there will be no confusing or conflicting code when the Mongolian control is used on different computers.

The operating system deals with all character information by using the internal code. The internal code actually is the stored code of each character in the computer and its function is to unify the expression of various character-input codes. Information-exchange code of Mongolian has a simple corresponding relation with the internal code, which can be easily found through the information-exchange codes of Mongolian.

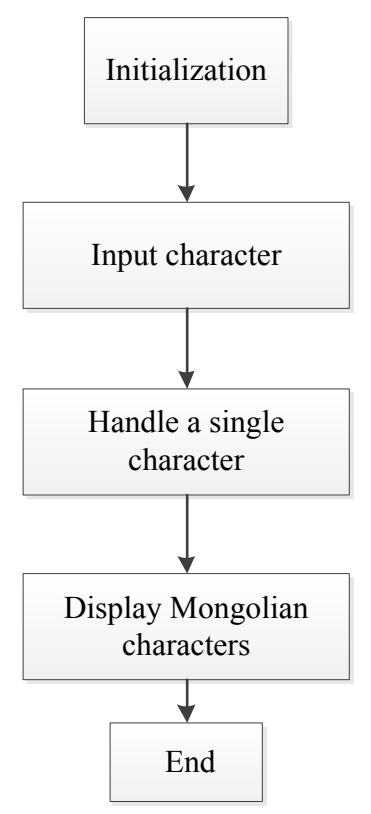

Fig. 8. Program design structure 
Mongolian input: Mongolian input is realized by utilizing the existing English keyboard, through which the input and mixed typesetting of Mongolian, Chinese and figures can be realized [16]. Input code is used in inputting. When inputting Mongolian, which character is input depends on which key is pressed, and the input code is in accordance with the code inside the computer. The corresponding list of Mongolian characters and English keyboard is shown in Figure 6.

Mongolian output: Font information of Mongolian literal pool is continuously stored in the storage medium in a certain order [17] while most of Mongolian address codes are continuous and ordered and have a simple corresponding relation with the Mongolian internal code. The Mongolian address code refers to the logical address storing of Mongolian font information in the Mongolian literal pool.

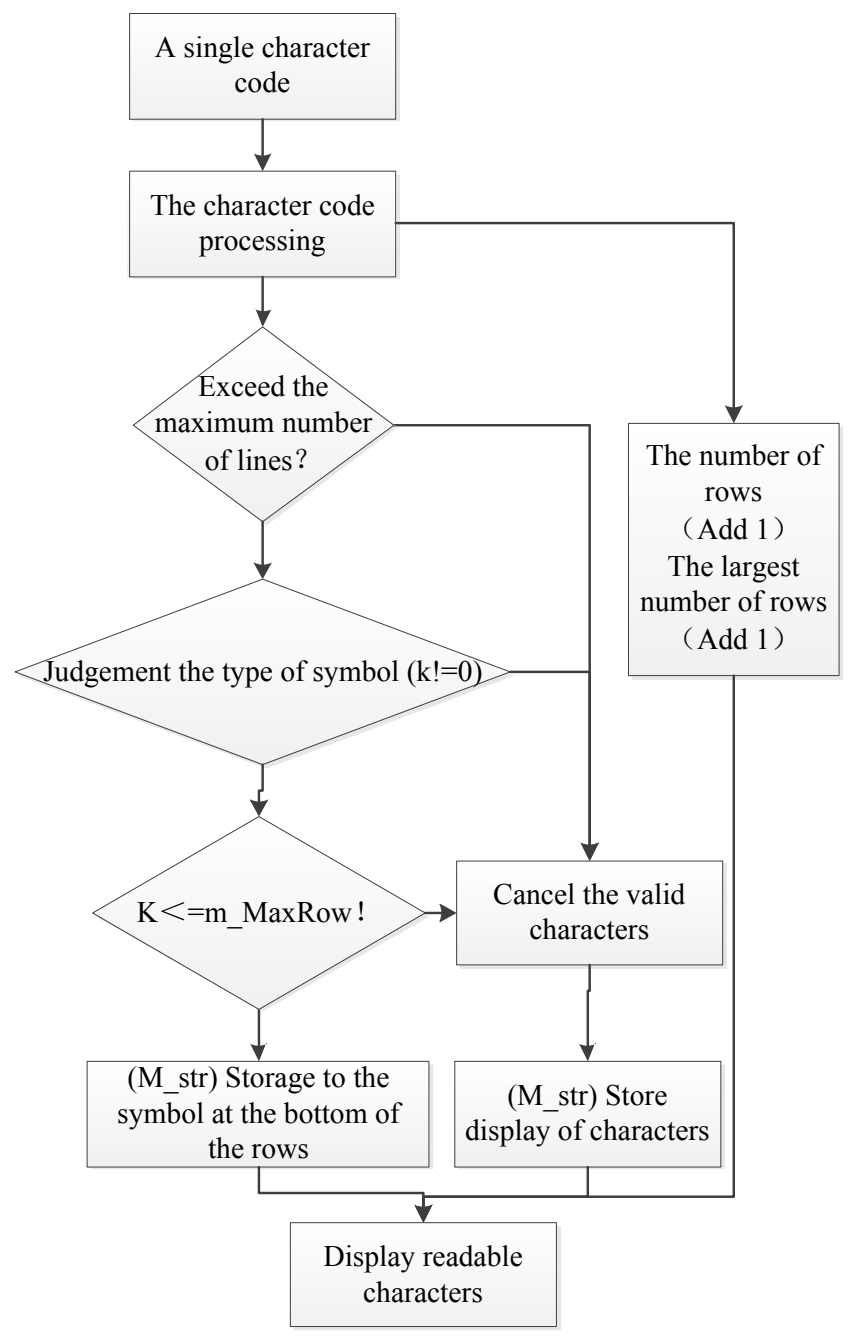

Fig. 9. A single character processing flow chart 
Mongolian processing procedure : The Mongolian code is the basis of Mongolian information processing and also is the precondition. The internal code is the code for character processing and it and the literal pool are the basic conditions for Mongolian information processing. The address code is used for character output with the dot matrix font of a character by the address code, and the character font can be displayed by utilizing the output equipment. These concepts are correlative with each other and jointly accomplish the whole process from the input, processing to the output. The processing procedure of Mongolian characters is shown in Figure 7.

\subsection{Mongolian controls process}

The making process of the Mongolian "text control", as shown in Figure 8 programming chart, is completely consistent. Processing a single character is shown in Figure 9.

ReadString () handles string $m_{-}$str part of the process, as shown in Figure 10.

Mongolian scrip displays from left to right. At the same time, the controls are able to implement mixed Mongolian and Chinese. Mongolian and Chinese have obvious differences in arrangement. When preparing the control option, priority is given to Mongolian. As a result, the string will show as a unit. Showing the whole process of text content requires constant calls to display text lines.

A text line uses the process DispCol (). Flow chart 11 DispCol () are as follows.

\section{$5 \quad$ Analysis and Discussion on the Test Results Analysis and Discussion for Teaching Software}

5.1 English ability discussion on experimental class and comparative class

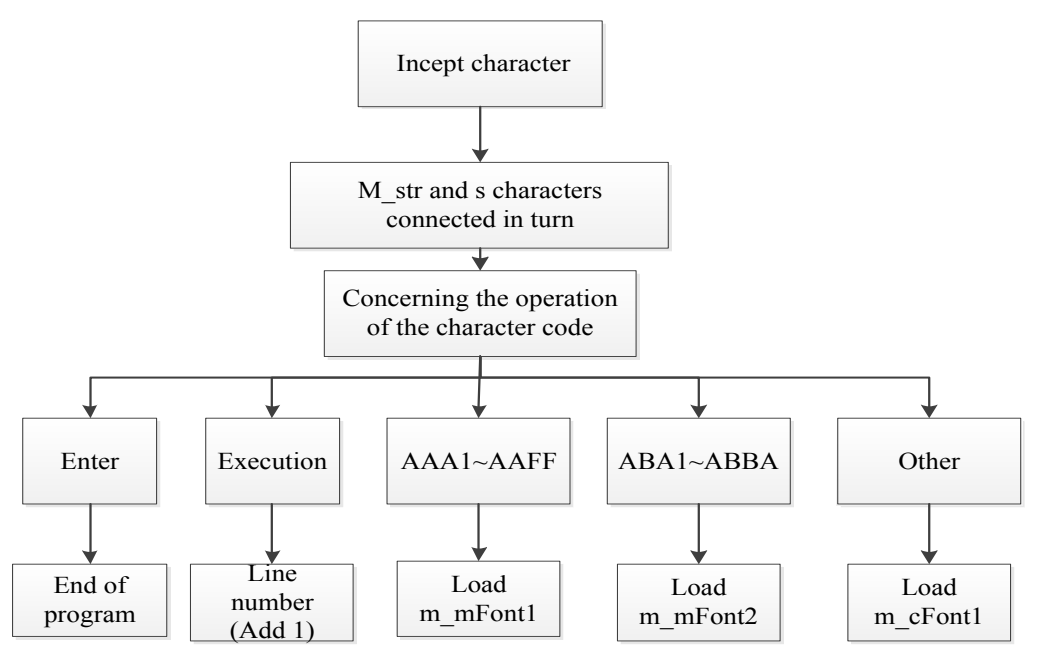

Fig. 10.Read String () function flow chart 


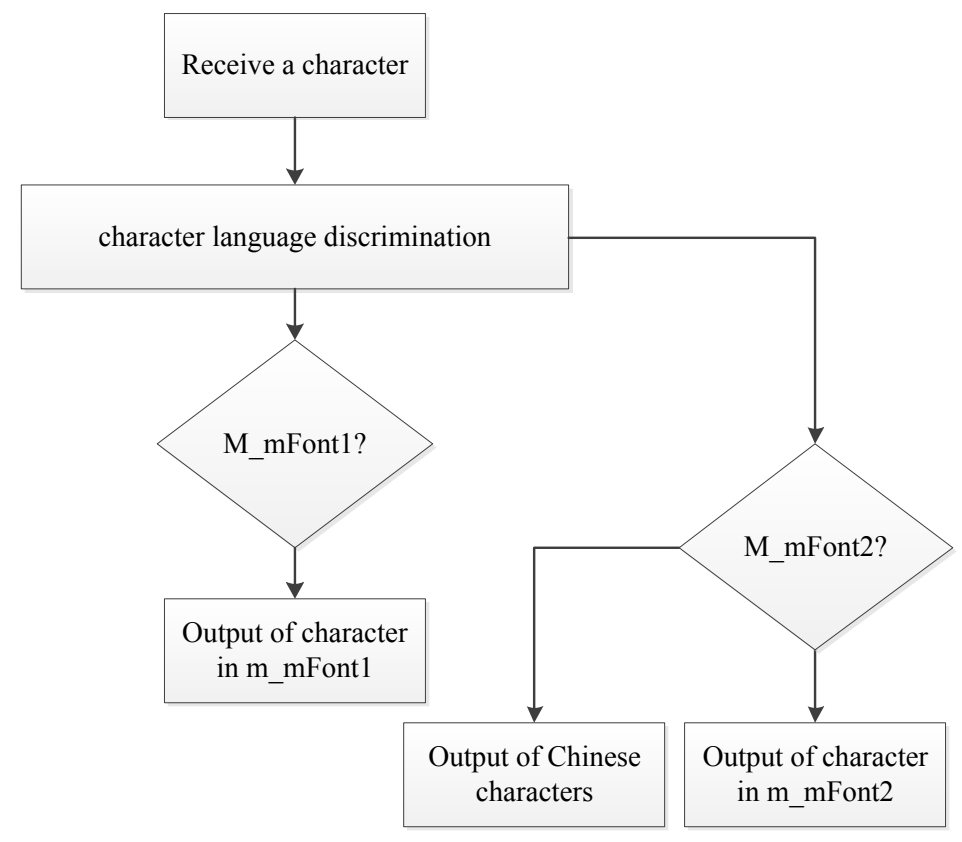

Fig. 11.Text line output flow chart

The post-test results in Table 1 show that the English average grades in both experimental class and comparative classes have improved, and reached the significant level. The average score in the experimental class climbs from 41.79 in pre-test to 62.73 in post-test, increasing by 20.94 . The average score in the comparative class goes up from 42.00 in pre-test to 53.51 in post-test, increasing by 11.51 .

Table 1. Test results contrast

\begin{tabular}{|c|c|c|c|c|c|c|c|c|}
\hline & \multicolumn{2}{|c|}{ Pre-test } & \multicolumn{2}{|c|}{ Post-test } & \multicolumn{2}{|c|}{$\begin{array}{c}\text { Difference before and } \\
\text { after }\end{array}$} & \multicolumn{2}{|c|}{ Inspection } \\
\hline & Mean & $S D$ & Mean & $S D$ & Mean & $S D$ & $T$ & $\boldsymbol{P}$ \\
\hline Experimental classes & 41.8 & 13.80 & 62.73 & 12.59 & -20.94 & 15.26 & -8.98 & $<0.001$ \\
\hline Comparative class & 42 & 9.15 & 53.51 & 11.29 & -11.50 & 15.28 & -10.56 & $<0.001$ \\
\hline $\mathrm{T}$ & \multicolumn{2}{|c|}{-0.85} & \multicolumn{2}{|c|}{-3.469} & \multicolumn{2}{|c|}{2.86} & & \\
\hline $\mathrm{P}$ & \multicolumn{2}{|c|}{$>.05$} & \multicolumn{2}{|c|}{$<.01$} & \multicolumn{2}{|c|}{$<.01$} & & \\
\hline
\end{tabular}

We can see that the improvement rate of the experimental class is 9.43 higher than that in the comparative class. It shows that, by using the teaching software, students' English ability has improved greatly in the experimental class.

\subsection{English skills analysis and discussion on three group}

In order to further analyze the influence of computer aided-teaching software on students with different levels of English ability. 38 tested students are divided into 
three groups according to the English language ability shown in the pre-test. The high-score group surpassed the average with a standard deviation reaching 7 . The low-score group scored lower than the average with a standard deviation amount to 9. 22 students are in the middle group. Through the comparison between groups' English average scores in the pre-test and post-test, we can determine their progress in English. The data obtained are carried out matching T test by SPSS13.0.

From the pre-test and post-test average results in high, medium and low groups in Table 2, we can see that the average score of pre-test in the low score group is 24.33 , and the post-test average score is 57.22 , up by 32.89 . The average score of pre-test in the high score group is 64.14 , and the post-test average score is 67.71 , up by 3.57 . The average score of pre-test in the medium score group is 41.82 , and the post-test average score is 63.32 , up by 21.50 . Although the three groups all have improved, the improvement of the low and medium score groups is obviously higher than that of the high score group, with the low score group ranking first in improvement. This indicates that the use of computer teaching software is most effective for the low score group of students, confirming the experimental results of Song Mahjong (1998). The multimedia teaching means are effective to improve the foreign language ability, and the level of impact of training is related to the level of the second language. Students with poor English ability can benefit much more from the new training than those who already have good English ability.

Table 2. Different grouping test scores contrast

\begin{tabular}{|l|c|c|c|}
\hline & Low score group & Medium score group & High score group \\
\hline Pre-test & 24.3 & 41.85 & 64.14 \\
\hline Post-test & 57.3 & 63.35 & 67.75 \\
\hline Difference & 32.5 & 21.65 & 3.56 \\
\hline $\mathrm{T}$ & 9.05 & 9.66 & 0.756 \\
\hline $\mathrm{P}$ & $<0.05$ & $<0.01$ & $>0.05$ \\
\hline
\end{tabular}

\section{Conclusion}

To solve the insufficiency of Mongolian college students in English learning, computer-assisted teaching software applicable to the Inner Mongolian students and teachers for learning and teaching bilingual language using Mongolian script is designed. The interface of the software is friendly with abundant content, and an intertranslation code based on ASCII code resolves the independent code of bilingual characters, Mongolian input and output, Mongolian character processing and other difficult problems. Development of this software plays a positive role in the efficient English learning of Inner Mongolian college students, and user feedback reveals that the feeling of using the software for the students is good. This software has a significant impact in improving English performance of students and assisting teachers in English teaching. 


\section{$7 \quad$ References}

[1] X. Tian, "Study on the computer-assisted English writing instruction", Advanced Materials Research, Vol. 1044-1045, pp. 1660-1663, 2014.

[2] L. Xiaoxia, W. Hongmei, "Research of computer-assisted english teaching system design based on constructivism", RISTI - Revista Iberica de Sistemas e Tecnologias de Informacao, Vol.17, pp.64-67, March 2016.

[3] Z. Xiaowei, W. Xiaoli, and W. Yan, "Analysis of reliability and validity in computer assisted English teaching test", Advanced Materials Research, Vol.984, pp.5029-5032, 2014.

[4] J. Yejun, Q. Jiahui, "The interaction design and realization of answer system of computerassisted English examination system based on QT WebKit", 2011 IEEE 3rd International Conference on Communication Software and Networks, ICCSN 2011, pp.5-8,2011.

[5] J. Jiyou, "CSIEC: A computer assisted English learning chatbot based on textual knowledge and reasoning”, Knowledge-Based Systems, Vol. 22, pp. 249-255, May 2009. https://doi.org/10.1016/j.knosys.2008.09.001

[6] C. Yuming, L. Chienyu, and L. Yikuan, "The preferences of young children for images used in dynamic graphical interfaces in computer-assisted English vocabulary learning", Displays, Vol.26, pp.147-152, October 2005. https://doi.org/10.1016/j.displa.2005.06.002

[7] C. You, "The application of multimedia computer assisted language learning in English teaching", Advanced Materials Research, vol.926, pp.4634-4637, 2014.

[8] Z. Fulin, Y. Mingqing, "Constructivism computer-assisted instruction model for college English study", Communications in Computer and Information Science, Vol.233, pp.200206,2011. https://doi.org/10.1007/978-3-642-24010-2 27

[9] L. Lin, "On correlation analysis of computer-assisted oral English test achievements with computer preference", Proceedings - 2013 International Conference on Computational and Information Sciences, ICCIS 2013, pp.1750-1753,2013. https://doi.org/10.1109/iccis. 2013.457

[10] B. Varsha, "Enhancing computer assisted language learning via the world wide web for English language teaching", Proceedings - IEEE International Conference on Technology for Education, T4E 2011, pp.291-292,2011.

[11] R. Sun, "Analysis of teacher role in computer-based English teaching," Communications in Computer and Information Science, Vol. 267, pp. 458-463, August 2012. https://doi.org/10.1007/978-3-642-29084-8 71

[12] D.L. Yang, Z. H., "Research on the framework of new college English teaching mode integrating cooperative and autonomous learning in the network multimedia environment". ICETC 2010-2010 2nd International Conference on Education Technology and Computer, Vol.3, pp. 3256-3259, 2010.

[13] S. Li, "Survey research on college students' English learning anxiety in the computer network environment". ICCSE 2011-6th International Conference on Computer Science and Education, Vol.36, pp.1010-1012, 2011.

[14] D.L. Yang, H. Zheng, "Research on the framework of new college English teaching mode integrating cooperative and autonomous learning in the network multimedia environment," ICETC 2010-2010 2nd International Conference on Education Technology and Computer, vol. 3, pp. 3256-3259, April 2010

[15] Y. Liang, T.T. Zheng, "Computer-assisted college English teaching under the perspective of "embodied philosophy"," 2011 International Conference on Multimedia Technology, ICMT 2011, pp. 3005-3007. https://doi.org/10.1109/ICMT.2011.6003095 
[16] Q.H. Yang, Research of college English teaching based on computer network technology. Lecture Notes in Electrical Engineering, Vol. 206, pp.375-382, June 2013. https://doi.org/10.1007/978-1-4471-4790-9 48

[17] R. Sun, Analysis of teacher role in computer-based English teaching. Communications in Computer and Information Science, Vol. 267, pp. 458-463, August 2012. https://doi.org/10.1007/978-3-642-29084-8 71

[18] M. Haitao, Computer-based student-centered classroom in English language teaching. BMEI 2011 - Proceedings 2011 International Conference on Business Management and Electronic Information, Vol. 2, pp. 714-717, June 2011. https://doi.org/10.1109/icbmei. 2011.5918012

[19] P. Zhang, "Analysis on the development of computer basic education under network environment". TEIN 2012-2012 3rd International Conference on Telecommunication and Information, vol.11, pp. 137-141, 2012.

\section{Author}

Xiaojun Zhao, born in 1971. He is a professor from Inner Mongolia Normal University, majors in curriculum and pedagogy.

The paper is part of national social science foundation "Research on formation path of Mongolian college students English effective learning from the trilingual perspective". Project number: BMA 140038. Article submitted 16 July 2016. Published as resubmitted by the author 10 October 2016. 